\title{
A generalization of Szebehely's inverse problem of dynamics
}

\author{
W. Sarlet ${ }^{a, b}$, T. Mestdag ${ }^{a}$ and G. Prince ${ }^{b}$ \\ ${ }^{a}$ Department of Mathematics, Ghent University \\ Krijgslaan 281, B-9000 Ghent, Belgium \\ ${ }^{b}$ Department of Mathematics and Statistics, La Trobe University \\ Melbourne, Victoria 3086, Australia
}

\begin{abstract}
The so-called inverse problem of dynamics is about constructing a potential for a given family of curves. We observe that there is a more general way of posing the problem by making use of ideas of another inverse problem, namely the inverse problem of the calculus of variations. We critically review and clarify different aspects of the current state of the art of the problem (mainly restricted to the case of planar curves), and then develop our more general approach.
\end{abstract}

Keywords: Szebehely's equation, inverse problem of dynamics, inverse problem of the calculus of variations

\section{Introduction}

There are two kinds of inverse problems which have received significant attention in the literature, both related to Lagrangian mechanics in some sense, yet seemingly living separate lives. One is the so-called inverse problem of the calculus of variations, where the issue is, as far as classical mechanics is concerned, to study under what circumstances a system of second-order ordinary differential equations can be derived from a variational principle. When the equations of motion are given in normal form, this question amounts to finding a suitable matrix multiplier which in the end will be the Hessian of the Lagrangian. The other inverse problem is often referred to as the inverse problem of dynamics and is, roughly speaking, as follows. Given a family of paths in configuration space, find a potential such that the corresponding classical Lagrangian system for a particle with unit mass admits the given family as part of its integral curves. This question seems to have popped up in the area of celestial mechanics, particularly after Szebehely launched it in the context of satellite observations in a much cited paper in 1974 [31]. Without a variational content, i.e. when the idea is to determine general admissible forces related to a given family of curves, this problem actually has a much older history, dating back for example to a paper by Dainelli of 1880 [16]. It is astonishing 
that, in the extensive literature since Szebehely, when the requirement is imposed that such forces should fit into a Lagrangian description, nobody ever approached the problem from the point of view of the general inverse problem of the calculus of variations: it has always been taken for granted that the equations should be variational without allowing for an extra multiplier. Expressed differently, it is assumed that the kinetic energy term of the Lagrangian under construction is going to come from the standard Euclidean metric. Our goal is to fill this gap and thus bring these two separate inverse problems closer together. To be on the safe side, however, we should say right away that there have been contributions in which a general Riemannian metric is used for the kinetic energy function (see e.g. [23], [5], [24]). But the idea in those papers is that one starts the formulation of the problem from a preassigned metric $g$, which is again altogether a different problem than the one we have in mind, where the metric in fact is part of the unknowns.

We give a brief sketch of the inverse problem of the calculus of variations for SODEs (second-order differential equations) in the next section. For a comprehensive survey of the inverse problem of dynamics in Section 3, we will limit ourselves here mostly to the case of two equations $(n=2)$. The reason is that there is a fundamental difference between $n=2$ and $n>2$ in this field, as we will briefly indicate in that section. Our generalization to allow for more general multipliers is discussed in Section 4. A number of examples in the subsequent section will illustrate that our generalization covers a much wider range of admissible potentials than those considered so far in the literature.

\section{The inverse problem of the calculus of variations for SODES}

It will be sufficient for our later purposes that we discuss only the situation for autonomous second-order differential equations. Let $\Gamma$ be the SODE vector field on a tangent bundle, modelling the general system of differential equations

$$
\ddot{x}^{i}=F^{i}(x, \dot{x}), \quad i=1, \ldots, n .
$$

The inverse problem of the calculus of variations is the search for a non-singular symmetric multiplier matrix $g_{i j}(x, \dot{x})$ such that

$$
g_{i j}\left(\ddot{x}^{j}-F^{j}\right) \equiv \frac{d}{d t}\left(\frac{\partial L}{\partial \dot{x}^{i}}\right)-\frac{\partial L}{\partial x^{i}},
$$


for some Lagrangian function $L(x, \dot{x})$. Necessary and sufficient conditions for this $g$, generally referred to as the Helmholtz conditions, are that

$$
\begin{aligned}
& \Gamma\left(g_{i j}\right)=g_{i k} \Gamma_{j}^{k}+g_{j k} \Gamma_{i}^{k}, \quad \Gamma_{j}^{i}:=-\frac{1}{2} \frac{\partial F^{i}}{\partial \dot{x}^{j}}, \\
& \frac{\partial g_{i j}}{\partial \dot{x}^{k}}=\frac{\partial g_{i k}}{\partial \dot{x}^{j}}, \\
& g_{i j} \Phi_{k}^{j}=g_{k j} \Phi_{i}^{j}, \quad \Phi_{j}^{i}:=-\frac{\partial F^{i}}{\partial x^{j}}-\Gamma_{k}^{i} \Gamma_{j}^{k}-\Gamma\left(\Gamma_{j}^{i}\right) .
\end{aligned}
$$

There is an extensive literature about this problem. We limit ourselves to citing only a few sources [30, 26, 4, 14, 18, 1, 22, 12, where the reader might appreciate the variety of analytical and geometric methods which have been used in this field.

For now, it is enough that we look at the more restrictive situation where $F^{i}=F^{i}(x)$ in the given coordinate description of $\Gamma$. It is then plausible that we restrict the search for a multiplier also to depend on the position variables only. In that case, the first condition (3) forces the $g_{i j}$ actually to be constant in those coordinates and all that remains is the simplified condition (5)

$$
g_{i j} \frac{\partial F^{j}}{\partial x^{k}}=g_{k j} \frac{\partial F^{j}}{\partial x^{i}}
$$

which of course is nothing but the integrability requirement expressing the existence of a potential function $V(x)$, such that

$$
g_{i j} F^{j}(x)=-\frac{\partial V}{\partial x^{i}}
$$

\section{The inverse problem of dynamics}

When surveying the vast literature in this field, one gets the impression that after the early papers on the subject, the situation has often been obscured by contributions in which the authors have paid insufficient attention to the sound practice of replacing at each step an original set of conditions by a new set which is (generically) equivalent to the previous one. This is in particular true for the confusion sometimes encountered about the role of the energy function in the story and about the number of partial differential equations for admissible potentials to be acquitted. It is therefore not a waste of time that we try to draw a clear picture of the overall situation before entering into our new generalization. As expressed before, the problem becomes quite different for $n>2$, so we will only discuss the case $n=2$ here.

The basic problem is this: given a family of paths in the form

$$
f(x, y)=c,
$$

find a potential $V(x, y)$ and a parameterisation such that the system

$$
\ddot{x}=-V_{x}, \quad \ddot{y}=-V_{y}
$$


has integral curves that are paths belonging to this family for suitable choices of initial conditions. Most cited in this area is the so-called Szebehely equation [31]:

$$
f_{x} V_{x}+f_{y} V_{y}+\frac{2(E-V)}{f_{x}^{2}+f_{y}^{2}}\left(2 f_{x} f_{y} f_{x y}-f_{x}^{2} f_{y y}-f_{y}^{2} f_{x x}\right)=0 .
$$

This is meant to be a first-order PDE for $V$, but what is $E$ ? Some papers say that $E$, which as usual represents the total energy of the system, must be given a pre-assigned constant value. Others say that it must be pre-assigned as some arbitrarily selected function of $f$. Bozis, one of the main contributors to the field, has rightly derived a second-order PDE for $V$ [7] which does not contain $E$, but he took the fact that $E=E(f)$ for granted to do that. It would be impossible to refer here to every other statement that has been made about such issues. But the reader can find a long list of references already in the review paper of Bozis [9], some less cited contributions are referred to in [27].

Without claiming originality in this section, we start our comprehensive overview of the situation by some general considerations. Let $Z$ be a vector field on $\mathbb{R}^{2}$ whose flow preserves the given function $f$, meaning that $Z(f)=0$. So $Z$ at each point of $f(x, y)=c$ represents the tangent direction to a curve of the family. As is well known, the complete lift of $Z$ to the tangent bundle $T \mathbb{R}^{2}$, i.e. the vector field

$$
Z^{c}=Z^{i}(x) \frac{\partial}{\partial x^{i}}+u^{j} \frac{\partial Z^{i}}{\partial x^{j}} \frac{\partial}{\partial u^{i}}
$$

is defined by the property that its flow is the tangent map of the flow of $Z$ (see e.g. [15]). It follows that the lifts of integral curves of $Z$ are integral curves of $Z^{c}$. The idea is that we want to construct a dynamical system, i.e. a SODE vector field

$$
\Gamma=u^{i} \frac{\partial}{\partial x^{i}}+F^{i} \frac{\partial}{\partial u^{i}}
$$

such that $Z^{c}$ and $\Gamma$ coincide on points in the image of $Z$. This will guarantee that the lifted integral curves of $Z$ belong to the set of integral curves of $\Gamma$ (which has the property that all its integral curves are lifted curves). The condition for that to happen is that

$$
\left.Z^{c}\right|_{\operatorname{Im} Z}=\left.\Gamma\right|_{\operatorname{Im} Z} \quad \Longleftrightarrow \quad F^{i}=Z^{j} \frac{\partial Z^{i}}{\partial x^{j}}=Z\left(Z^{i}\right) .
$$

Since $Z\left(Z^{i}\right)$ is a function of the $x^{i}$ only, it suffices to look for forces $F^{i}$ which have the same property in the given coordinates. But more general forces could be allowed provided we write $\left.F^{i}\right|_{\operatorname{Im} Z}$ in (10).

A vector field $Z$ for which $Z(f)=0$ is determined up to an arbitrary factor $h(x, y)$ corresponding to re-parametrization, say

$$
Z=h Z_{0}=h\left(f_{y} \frac{\partial}{\partial x}-f_{x} \frac{\partial}{\partial y}\right)
$$


The extra condition which comes in now is that we want the SODE $\Gamma$ to correspond to a conservative system with potential $V$ and standard (Euclidian) kinetic energy. In other words, we require that

$$
\begin{aligned}
& Z\left(Z^{1}\right)=Z(h) f_{y}+h Z\left(f_{y}\right)=-V_{x} \\
& Z\left(Z^{2}\right)=-Z(h) f_{x}-h Z\left(f_{x}\right)=-V_{y} .
\end{aligned}
$$

The essential picture then clearly reads as follows: if $Z(h)$ is eliminated between these two equations, we obtain an algebraic relation for $h^{2}$, which when substituted back in any of the above equations produces a second-order PDE for $V$. Whenever a solution for $V$ of this PDE is obtained, $h^{2}$ will be determined and we have an admissible SODE $\Gamma$. So far, there seems to be no relation with the energy function or with Szebehely's equation. However, it is more appropriate to execute the above process in a slightly different and more efficient way. Eliminating $Z(h)$ is like multiplying (12) with $-Z(y)$ and adding the product of (13) with $Z(x)$. A system which is algebraically equivalent with (12, 13) then follows if we also multiply (12) with $Z(x)$ and take the sum with (13) multiplied by $Z(y)$. The resulting equivalent system reads

$$
\begin{aligned}
h^{2}\left(f_{x} Z_{0}\left(f_{y}\right)-f_{y} Z_{0}\left(f_{x}\right)\right) & =-\left(f_{x} V_{x}+f_{y} V_{y}\right), \\
Z\left(\frac{1}{2} h^{2}\left(f_{x}^{2}+f_{y}^{2}\right)+V\right) & =0 .
\end{aligned}
$$

Again, solving (14) for $h^{2}$ and substituting in (15) produces the second-order PDE for $V$ generated by Bozis. But (15) indicates the first integration of this PDE. The energy $E$ is of course a function on $T \mathbb{R}^{2}$, but we need its restriction

$$
\bar{E}:=\left.E\right|_{\operatorname{Im} Z}=\frac{1}{2} h^{2}\left(f_{x}^{2}+f_{y}^{2}\right)+V .
$$

Then (15) implies that $\bar{E}$ must be a function of $f$. Expressed differently, a first integration of the second-order PDE for $V$ yields the first-order Szebehely equation (9) in which the term $E$ must be interpreted as an arbitrary function $\bar{E}(f)$. Integrating this linear firstorder PDE will then introduce a second arbitrary function, $\sigma(\tilde{f})$ say. In fact, it is clear from the first-order terms in (9) that the function $\tilde{f}$ is such that $\tilde{Z}(\tilde{f})=0$, where $\tilde{Z}$ is any vector field of the form

$$
\tilde{Z}=\tilde{h} \tilde{Z}_{0}=\tilde{h}\left(f_{x} \frac{\partial}{\partial x}+f_{y} \frac{\partial}{\partial y}\right)
$$

Note that $Z$ and $\tilde{Z}$ are orthogonal with respect to the standard Euclidean metric, i.e. we have $Z^{1} \tilde{Z}^{1}+Z^{2} \tilde{Z}^{2}=0$. One can further exploit the freedom in $h$ and $\tilde{h}$ to make them commute. This explains why Broucke and Lass ([1] ) were able to obtain an expression for the general solution for $V$ in terms of orthogonal coordinates (see also [25], [29]).

A few comments are in order here concerning the case of higher dimension $n$. A given family of curves can be specified by $n-1$ relations of the form $f_{a}(x)=c_{a}$. The arguments about a dynamical system $\Gamma$ satisfying a condition of the form (10) remain the same and give rise to a set of $n$ conditions such as $(12,13)$. This time, elimination of $Z(h)$ gives 
rise to $n-1$ algebraic expressions for $h^{2}$, supplemented by a single extra condition such as (15). Compatibility between the $n-1$ relations for $h^{2}$, however, creates by itself $n-2$ first-order PDEs for $V$, which is an entirely different story. We will discuss the case $n>2$ in a subsequent paper. As an aside, there is also a potentially interesting link with a form of generalized Hamilton-Jacobi theory. Indeed, the reasoning which led us to condition (10), if abstraction is made of a given family of curves and $Z$ therefore is any given vector field on the base manifold, is precisely what characterizes solutions of the generalized Lagrangian Hamilton-Jacobi problem as introduced in [13. For an early discussion of a link with the Hamilton-Jacobi problem, see [28].

To complete this section, we still have to report on a slightly different approach to the same problem, which is more adapted to a particular type of application. Going back to the requirement (10), when we first abstain from further restrictions on the forces and so allow for non-conservative forces with components $X$ and $Y$, these are simply given by the left-hand sides of (12,13). In some papers, a first-order PDE for $X$ and $Y$ is set up to study 'admissible non-conservative forces' (see e.g. [6] and [3]). For the sake of completeness, we will come back to this point at the end of this section. For now, however, setting up such a PDE seems an unnecessary complication because the left-hand sides of $(12,13)$ simply give the general expression of such admissible forces in terms of an arbitrary function $h$. In fact, these are more or less the expressions discussed in [16], but it looks better to write them as functions of say $\eta=h^{2}$ and its derivatives. Explicitly then they read,

$$
\begin{aligned}
2 X & =\eta_{x} f_{y}^{2}-\eta_{y} f_{x} f_{y}+2 \eta\left(f_{y} f_{x y}-f_{x} f_{y y}\right), \\
2 Y & =-\eta_{x} f_{x} f_{y}+\eta_{y} f_{x}^{2}+2 \eta\left(f_{x} f_{x y}-f_{y} f_{x x}\right) .
\end{aligned}
$$

We can subsequently impose the requirement that the forces derive from a potential, i.e. should be of gradient type. The necessary and sufficient condition

$$
X_{y}=Y_{x},
$$

now leads to a second-order PDE for $\eta$ and every solution will directly lead to an admissible potential by quadratures. That this version of the problem is equivalent to the one for obtaining $V$ through Szebehely's equation was demonstrated in [17, at least taking for granted that we already know that $\bar{E}$ will be some function of $f$. We will give a somewhat different interpretation to a calculation which can be found in that context in [17]. Considering (9) for $V$, with $E$ replaced by $\bar{E}(f)$, it is natural to replace $V$ by the function $2(\bar{E}-V) /\left(f_{x}^{2}+f_{y}^{2}\right)$. This does indeed simplify the equation for $V$. But this new dependent variable is after all $\eta=h^{2}$. The transformed equation is

$$
f_{x} \eta_{x}+f_{y} \eta_{y}+2 \eta\left(f_{x x}+f_{y y}\right)=2 \bar{E}^{\prime}(f) .
$$

This can only be an integrated form of the second-order PDE for $\eta$ resulting from (20), an integrated form which again has introduced an arbitrary function of $f$. In other words, if we act with $Z$ or $Z_{0}$ on (21), the resulting second-order PDE will be the same as the one following from (20). 
Finally, we touch upon an important point which is rarely explained in the vast literature on the subject. By construction (see (10)) it is only on $\operatorname{Im} Z$ that our dynamical system will have integral curves which are lifts of curves belonging to the given family. Moreover, the 2-dimensional submanifold $\operatorname{Im} Z$ of $T \mathbb{R}^{2}$ lies on some 3-dimensional hypersurface of constant energy. So not all solutions of the SODE we constructed will, by far, correspond to the curves we started from. To pin down solutions which do have this property, the initial conditions $\left(x_{0}, y_{0}, \dot{x}_{0}, \dot{y}_{0}\right)$ have to be prescribed as follows. Starting from an arbitrary initial position $\left(x_{0}, y_{0}\right), f\left(x_{0}, y_{0}\right)$ will fix a constant $c_{0}$. There is then no more freedom in selecting an initial velocity. Indeed, for every particular solution for an admissible $V, h^{2}$ will be fixed, by (14) for example, and $\bar{E}$ as defined by (16) will be a specific function of $f$, whose constant value $E_{0}$ therefore is fixed by $c_{0}$. Hence, the admissible initial velocity $\left(\dot{x}_{0}, \dot{y}_{0}\right)$ is completely determined by the requirements that it must give a tangent direction to $f$ at the point $\left(x_{0}, y_{0}\right)$ and satisfy the relation $\frac{1}{2}\left(\dot{x}_{0}^{2}+\right.$ $\left.\dot{y}_{0}^{2}\right)+V\left(x_{0}, y_{0}\right)=E_{0}$. This is in contrast with a series of older papers by Kasner (see [19] in the first place, and related work in [20] and [21]), which are only remotely related to the Szebehely problem, but have been referred to in passing in [9, 2] and [3].

Kasner [19] studied extensively the geometry of an orbit $y=y(x)$ which arises from the solution of a Newtonian system such as $\ddot{x}=X(x, y), \ddot{y}=Y(x, y)$. In such a set-up clearly there are three initial values which can be assigned arbitrarily, namely $x_{0}, \dot{x}_{0}$ and $\dot{y}_{0}$. Kasner shows that such an orbit satisfies a third-order ODE, which reads

$$
y^{\prime \prime \prime}\left(Y-y^{\prime} X\right)=y^{\prime \prime}\left(Y_{x}+y^{\prime}\left(Y_{y}-X_{x}\right)-y^{\prime 2} X_{y}\right)-3 y^{\prime \prime 2} X .
$$

The inverse problem that he poses and solves is about pinning down geometrical characteristics of curves which are also sufficient for orbits $y(x)$ to be generated by a Newtonian system. Clearly, this is a different matter. But for the benefit of the review features of this section, we can establish a link between Kasner's equation (22) and our approach to Szebehely's problem for the case that the orbit $y(x)$ under consideration is actually one which belongs to a given family $f(x, y)=c$. For that we go back to the equations (12, 13) and repeat the steps which led to the equivalent system (14, 15) when the right-hand sides are general functions $X(x, y)$ and $Y(x, y)$, not necessarily coming from a potential. The result is

$$
\begin{aligned}
h^{2}\left(f_{x} Z_{0}\left(f_{y}\right)-f_{y} Z_{0}\left(f_{x}\right)\right) & =f_{x} X+f_{y} Y, \\
Z_{0}\left(\frac{1}{2} h^{2}\left(f_{x}^{2}+f_{y}^{2}\right)\right) & =f_{y} X-f_{x} Y .
\end{aligned}
$$

Solving (23) for $\eta=h^{2}$ we get

$$
f_{x} X+f_{y} Y=\eta\left(2 f_{x} f_{y} f_{x y}-f_{x}^{2} f_{y y}-f_{y}^{2} f_{x x}\right),
$$

and substitution of this result in (24) gives

$$
Z_{0}\left(\frac{1}{2} \frac{f_{x}^{2}+f_{y}^{2}}{2 f_{x} f_{y} f_{x y}-f_{x}^{2} f_{y y}-f_{y}^{2} f_{x x}}\left(f_{x} X+f_{y} Y\right)\right)=f_{y} X-f_{x} Y
$$


This is the first-order PDE for $X$ and $Y$ referred to before, which we will give a different interpretation now. Let $y(x)$ be an orbit belonging to the given family, then $f(x, y(x)) \equiv$ c. A first differentiation determines $y^{\prime}$ by $f_{x}+y^{\prime} f_{y}=0$ and a further differentiation provides a formula for $y^{\prime \prime}$. In addition, along the curve $y(x)$, we have that $Z_{0}=f_{y} d / d x$. This way, it follows from (26) that

$$
\frac{d}{d x}\left(\frac{1}{2} \frac{1+y^{\prime 2}}{y^{\prime \prime}}\left(Y-y^{\prime} X\right)\right)=X+y^{\prime} X
$$

This is a third-order ODE for $y(x)$ which indeed is Kasner's equation (22). It is worthwhile observing that also this equation can be integrated once in the case that the forces derive from a potential. Indeed, in such a case we have that $X+y^{\prime} Y=-d V / d x$ and (27) implies that

$$
(e-V) y^{\prime \prime}=\frac{1}{2}\left(1+y^{\prime 2}\right)\left(y^{\prime} V_{x}-V_{y}\right),
$$

where $e$ is an integration constant which of course relates again to the energy integral of the system.

\section{The amalgamated inverse problem}

Looking back at the various features of the Szebehely problem as summarized so far, it seems to us that the process of determining admissible forces $(X(x, y), Y(x, y))$ for a given family of paths $f(x, y)=c$ is very straightforward: there is not even a problem there, as the expressions (18, 19) provide a wealth of possibilities, in terms of an arbitrary function $\eta$. Restrictions come in when we require that such forces arise from a variational principle. But as we asked in the introduction, why then should a Lagrangian for the system be restricted to have a standard Euclidean kinetic energy? As explained in Section 2, if we allow a symmetric, non-singular multiplier $g_{i j}(x, y)$, it must be constant. That still means, however, that the condition (20) of the previous section can be relaxed to

$$
g_{12}\left(Y_{y}-X_{x}\right)=g_{22} Y_{x}-g_{11} X_{y} .
$$

This creates a more general second-order PDE for $\eta=h^{2}$, containing three extra parameters which can make the difference in certain applications. We will illustrate this with an extensive example in the next section. Every solution for $\eta$ of (29) will as before directly lead to an admissible potential by quadratures, because (29) simply guarantees integrability of the system

$$
\begin{aligned}
& g_{11} X+g_{12} Y=-V_{x}, \\
& g_{12} X+g_{22} Y=-V_{y},
\end{aligned}
$$

for $V$, leading to a Lagrangian of the form $L=\frac{1}{2} g_{i j} \dot{x}^{i} \dot{x}^{j}-V$. For now, it remains to explain how the other aspects of the theory discussed in the previous section generalize here, in particular with respect to the Szebehely equation. An observation made with

the introduction of the vector field $\tilde{Z}_{0}$ in (17) indicates how to proceed. The point is 
that passing from the original conditions (12, 13) to the equivalent set (14, 15) can be seen as coming from the action on $V$ with the two orthogonal vector fields $\tilde{Z}_{0}$ and $Z_{0}$. With the vector field $Z$ still as in (11), the requirements (30,31) can be written in index notation as

$$
g_{i j} Z\left(Z^{j}\right)=-V_{x^{i}}, \quad \text { with } \quad Z\left(Z^{j}\right)=h Z_{0}(h) Z_{0}^{j}+h^{2} Z_{0}\left(Z_{0}^{j}\right) .
$$

Observe that the term involving $Z(h)=h Z_{0}(h)$ has coefficient $g_{i j} Z_{0}^{j}$, i.e. comes from the contraction of the metric $g$ with the vector field $Z_{0}$. The process of elimination of $Z(h)$ between the two equations therefore amounts to taking a further contraction with the orthogonal vector field

$$
Z_{0}^{\perp}:=\left(g_{22} f_{x}-g_{12} f_{y}\right) \frac{\partial}{\partial x}+\left(g_{11} f_{y}-g_{12} f_{x}\right) \frac{\partial}{\partial y},
$$

defined (up to a factor) by $g\left(Z_{0}, Z_{0}^{\perp}\right)=0$. The term involving $h^{2}$ on the other hand has coefficient $g_{i j} Z_{0}\left(Z_{0}^{j}\right)$. Now $Z_{0}\left(Z_{0}^{j}\right)$ makes perfectly sense as components of a vector field in the given coordinates. Indeed, since our metric $g$ is constant in the given coordinates, the connection coefficients of the Levi-Civita connection $\nabla$ are zero and $Z_{0}\left(Z_{0}^{j}\right)$ are the components of $\nabla_{Z_{0}} Z_{0}$. Explicitly, we have

$$
\nabla_{Z_{0}} Z_{0}=Z_{0}\left(f_{y}\right) \frac{\partial}{\partial x}-Z_{0}\left(f_{x}\right) \frac{\partial}{\partial y}
$$

The last point to observe is that multiplication of (32) with $Z_{0}^{i}$ (and summation) makes the left-hand side combine into $Z_{0}\left(\frac{1}{2} h^{2} g_{i j} Z_{0}^{i} Z_{0}^{j}\right)$. In conclusion, proceeding in exactly the same way as in the previous section, the equivalent set of conditions which generalizes (14, 15) will read now

$$
\begin{array}{r}
h^{2} g\left(\nabla_{Z_{0}} Z_{0}, Z_{0}^{\perp}\right)=-Z_{0}^{\perp}(V), \\
Z_{0}\left(\frac{1}{2} h^{2} g_{i j} Z_{0}^{i} Z_{0}^{j}+V\right)=0 .
\end{array}
$$

As before, solving (35) for $h^{2}$ and substituting into (36) gives rise to a second-order PDE for $V$, this time involving three extra parameters $g_{i j}$ as yet to be determined. Again, (36) indicates that the energy function $E:=\frac{1}{2} g_{i j} \dot{x}^{i} \dot{x}^{j}+V$ restricted to $\operatorname{Im} Z$ is a function of $f$ :

$$
\left.E\right|_{\operatorname{Im} Z}=\bar{E}(f) \text {. }
$$

As a result, the afore-mentioned second-order PDE integrates to a first-order equation which can be written in the form

$$
Z_{0}^{\perp}(V)+\frac{2(\bar{E}-V)}{g\left(Z_{0}, Z_{0}\right)} g\left(\nabla_{Z_{0}} Z_{0}, Z_{0}^{\perp}\right)=0 .
$$

This is the corresponding generalized Szebehely equation. Explicitly, for comparison with the original Szebehely equation (9),

$$
Z_{0}^{\perp}(V)+\frac{2(\bar{E}-V)(\operatorname{det} g)}{g_{11} f_{y}^{2}-2 g_{12} f_{x} f_{y}+g_{22} f_{x}^{2}}\left(2 f_{x} f_{y} f_{x y}-f_{x}^{2} f_{y y}-f_{y}^{2} f_{x x}\right)=0 .
$$


The link between this equation and the second-order equation (29) for $\eta$ also works in the same way as in the previous section. It suffices to introduce $2(\bar{E}-V) / g\left(Z_{0}, Z_{0}\right)$, which by the energy relation is simply our function $\eta=h^{2}$, as new dependent variable in (38). Then

$$
\begin{aligned}
Z_{0}^{\perp}(\eta) & =\frac{2}{g\left(Z_{0}, Z_{0}\right)}\left(\bar{E}^{\prime}(f) Z_{0}^{\perp}(f)-Z_{0}^{\perp}(V)\right)-\frac{2(\bar{E}-V)}{g\left(Z_{0}, Z_{0}\right)^{2}} 2 g\left(\nabla_{Z_{0}^{\perp}} Z_{0}, Z_{0}\right), \\
& =\frac{2}{g\left(Z_{0}, Z_{0}\right)}\left(\bar{E}^{\prime}(f) Z_{0}^{\perp}(f)+\eta g\left(\nabla_{Z_{0}} Z_{0}, Z_{0}^{\perp}\right)-\eta g\left(\nabla_{Z_{0}^{\perp}} Z_{0}, Z_{0}\right)\right)
\end{aligned}
$$

where we used equation (38) for replacing $Z_{0}^{\perp}(V)$ on the right. It so happens that $g\left(Z_{0}, Z_{0}\right)=Z_{0}^{\perp}(f)$ and we know that $g\left(\nabla_{Z_{0}} Z_{0}, Z_{0}^{\perp}\right)=-g\left(\nabla_{Z_{0}} Z_{0}^{\perp}, Z_{0}\right)$. This way, the transformed equation can be written as

$$
Z_{0}^{\perp}(\eta)+2 \eta \frac{g\left(\nabla_{Z_{0}} Z_{0}^{\perp}+\nabla_{Z_{0}^{\perp}} Z_{0}, Z_{0}\right)}{g\left(Z_{0}, Z_{0}\right)}=2 \bar{E}^{\prime}(f) .
$$

It is further straightforward to compute that

$$
\nabla_{Z_{0}} Z_{0}^{\perp}+\nabla_{Z_{0}^{\perp}} Z_{0}=\left(g_{22} f_{x x}-2 g_{12} f_{x y}+g_{11} f_{y y}\right) Z_{0} .
$$

It follows that the above equation for $\eta$ takes the fairly simple form

$$
Z_{0}^{\perp}(\eta)+2 \eta\left(g_{22} f_{x x}-2 g_{12} f_{x y}+g_{11} f_{y y}\right)=2 \bar{E}^{\prime}(f) .
$$

As before, since the right-hand side vanishes under the action of $Z_{0}$, acting with $Z_{0}$ on the left will produce exactly the second-order PDE for $\eta$ which follows from (29).

The discussion in the previous section about admissible initial conditions remains the same here, with of course an adapted expression for the energy function $E$.

No doubt some of the results reported in this section, in particular the generalized Szebehely equation, must correspond to a particular case for constant $g$ in papers where the analysis starts from a pre-assigned Riemannian metric. Indeed it can be verified that the Szebehely type equation for "a particle describing orbits on a given surface" derived in [24] reduces identically to (39) when the $g_{i j}$ are taken to be constant.

In the next section, we want to explore to what extent our generalization opens up new possibilities for admissible potentials. The best thing to do then is to look at applications in which the authors have looked for the existence of a potential within a class of admissible forces, satisfying a particular ansatz. In such a case, the best starting point is simply the condition (29) on the forces $X$ and $Y$. It is then of interest to know that there exists a linear relation between $X, Y$ and the function $\eta$, namely the relation (25). Hence, with given $f$ and an ansatz about the form of $X$ and $Y$, the best strategy to adopt is the following. First use (25) to learn about the effect of the ansatz on $\eta$, and substitute this information back into one of the general expressions (18, 19) for $X$ and $Y$. This will immediately lead to restrictions on the forces, leading in general to a 
number of different case studies. For each of the subcases identified in the previous step, imposing the inverse problem condition (29) then should lead to further subcases, for which a non-singular, constant multiplier $\left(g_{i j}\right)$ exists, with corresponding potential $V$. Incidentally, recalling that $\eta$ actually stands for $h^{2}$, the information coming from (25) has led people to introduce what they called 'family boundary curves', which essentially determine from the requirement that $\eta$ should be positive the boundary of domains in $\mathbb{R}^{2}$ where admissible forces of a certain type exist (see e.g. [8]).

\section{$5 \quad$ Examples}

1. We start with the toy example of families of straight lines. It is quite trivial to verify that infinitely many potentials for such a family exist already in the classical picture with the standard Euclidean metric (see e.g. [10]), so there is no real need for a generalization here. But it is instructive to see how the generalization works anyway. Let us choose the coordinate axes in such a way that the straight lines are parallel to the $x$-axis, so that $f(x, y) \equiv y=c$. The general expressions (18,19) for admissible forces reduce to

$$
2 X=\eta_{x}, \quad Y=0 .
$$

We can then right away impose the inverse problem condition (29), which reads $g_{12} \eta_{x x}=$ $g_{11} \eta_{x y}$ and integrates to

$$
g_{11} \eta_{y}-g_{12} \eta_{x}=\phi(y), \quad \phi \text { arbitrary }
$$

This is in fact the equation (41) for this case and it further integrates to

$$
\eta=\sigma(y)+\psi\left(g_{11} x+g_{12} y\right)
$$

with $\sigma$ and $\psi$ arbitrary functions of the indicated arguments. Now $X$ becomes $X=$ $\frac{1}{2} g_{11} \psi^{\prime}$ and it readily follows from (30, 31) that $V=-\frac{1}{2} g_{11} \psi$. Hence we have a threeparameter family of kinetic energy functions and all admissible Lagrangians are of the form

$$
L=\frac{1}{2}\left(g_{11} \dot{x}^{2}+2 g_{12} \dot{x} \dot{y}+g_{22} \dot{y}^{2}\right)+\frac{1}{2} g_{11} \psi\left(g_{11} x+g_{12} y\right) \text {. }
$$

2. For a more instructive example, take

$$
f(x, y):=x y^{m}, \quad m \neq 0, m \neq-1 .
$$

Bozis [8] has carried out a comprehensive analysis about the existence of a potential for this $f$, starting from the ansatz that the forces should contain only terms which are quadratic and cubic in $x$ and $y$. He concluded that there are only two favourable situations then: one in which $m$ can be kept unspecified, but both force components then only contain two cubic terms and only the coefficient of $y^{2} x$ in $X$ can be left arbitrary; in the other favourable case $X$ and $Y$ have a quadratic and two cubic terms but $m=2$ 
(see also [9]). The values $m=0$ and $m=-1$ are excluded to avoid straight lines. Bozis actually missed a few cases in his analysis, but anyhow, we will see that within the same category of admissible forces, our generalization allows for many more favourable situations.

Say we put

$$
\begin{aligned}
& X=b_{1} y^{3}+b_{2} y^{2} x+b_{3} y x^{2}+b_{4} x^{3}+a_{1} y^{2}+a_{2} y x+a_{3} x^{2} \\
& Y=r_{1} y^{3}+r_{2} y^{2} x+r_{3} y x^{2}+r_{4} x^{3}+s_{1} y^{2}+s_{2} y x+s_{3} x^{2} .
\end{aligned}
$$

It follows from the relation (25) that for the given $f$,

$$
y X+m x Y=m(m+1) x y^{2 m-1} \eta .
$$

For using this information about $\eta$ into the general expression (18) for $X$, it is computationally appropriate to multiply both sides of (18) with $(m+1) y$. The resulting polynomial $2(m+1) y X$ on the left then has to match the following expression:

$$
\begin{aligned}
& (m-3) b_{1} y^{4}+2(m-1)\left(b_{2}+m r_{1}\right) y^{3} x+(3 m-1)\left(b_{3}+m r_{2}\right) y^{2} x^{2} \\
& \quad+4 m\left(b_{4}+m r_{3}\right) y x^{3}+m(5 m+1) r_{4} x^{4}+(m-2) a_{1} y^{3} \\
& \quad+(2 m-1)\left(a_{2}+m s_{1}\right) y^{2} x+3 m\left(a_{3}+m s_{2}\right) y x^{2}+m(4 m+1) s_{3} x^{3} .
\end{aligned}
$$

Immediately, from the coefficients of $x^{4}$ and $x^{3}$, it is clear that we must be in one of the following three cases:

Case 1: $\quad 5 m+1=0$ and $s_{3}=0$,

Case 2: $\quad 4 m+1=0$ and $r_{4}=0$,

Case 3: $\quad r_{4}=s_{3}=0$.

Similar information comes from the other end of the polynomials, i.e. identification of the coefficients of $y^{4}$ and $y^{3}$ shows that we must be in one of the following three cases:

Case a: $\quad m+5=0$ and $a_{1}=0$,

Case b: $m+4=0$ and $b_{1}=0$,

Case c: $\quad a_{1}=b_{1}=0$.

In addition, further identifications of coefficients require that

$$
\begin{aligned}
2 b_{2} & =(m-1) m r_{1}, \\
(3-m) b_{3} & =m(3 m-1) r_{2}, \\
(1-m) b_{4} & =2 m^{2} r_{3}, \\
3 a_{2} & =m(2 m-1) s_{1}, \\
(2-m) a_{3} & =3 m^{2} s_{2} .
\end{aligned}
$$


What follows is an elementary but rather tedious analysis of possible combinations. For each subcase of admissible forces we can identify, we subsequently impose the inverse problem condition (29) to see what further restrictions follow from the requirement of existence of a potential. We will not give details of all these calculations, but try to summarize the results in an appendix. By way of example, here comes a brief discussion of Case 1.

Case 1 requires Case $c$ and the further restrictions (43) mean that altogether we will have

$$
\begin{gathered}
m=-1 / 5, \quad s_{3}=0, \quad a_{1}=b_{1}=0, \quad r_{4} \text { so far arbitrary } \\
r_{1}=(25 / 3) b_{2}, \quad r_{2}=10 b_{3}, \quad r_{3}=15 b_{4}, \quad s_{1}=(75 / 7) a_{2}, \quad s_{2}=(55 / 3) a_{3} .
\end{gathered}
$$

Imposing (29) yields five more relations, this time involving the three extra parameters $g_{i j}$. At this point, we will not engage into an exhaustive analysis of all possible subcases, but try to look separately at solutions for a diagonal $g$ (not necessarily the unit matrix) and those with a non-diagonal $g$. With the choice $g_{12}=0$, we are further limited to $a_{2}=a_{3}=b_{3}=r_{4}=0$. The remaining free coefficients are $b_{2}$ and $b_{4}$, with corresponding values for $r_{1}$ and $r_{3}$, and since $g$ is determined up to an overall factor, we can for example take $g_{22}=1$ which then fixes $g_{11}=15\left(b_{4} / b_{2}\right)$. The potential is

$$
V(x, y)=-\frac{25}{12} b_{2} y^{4}-\frac{15}{2} b_{4} x^{2} y^{2}-\frac{15}{4} \frac{b_{4}^{2}}{b_{2}} x^{4} .
$$

With the choice $g_{12} \neq 0$, say $g_{12}=1$ without loss of generality, there is some more freedom in the coefficients of the expression for $X$. For example, $b_{2}, a_{2}$ and $a_{3}$ can be chosen arbitrarily; there are then conditions coming from (43) which will fix $b_{3}, b_{4}$ and $r_{4}$, and also $g_{11}$ and $g_{22}$ and the potential can be readily computed.

The conclusions which can be drawn from the more complete analysis in the appendix is that our generalization clearly identifies more general forces for which a potential exists in relation to the family $x y^{m}=c$ with unspecified $m$. In addition, we find specific solutions also for the following list of special values for $m$ (in order of appearance in our discussion): $-1 / 5,-1 / 4,-5,-4,-2 / 3,-1 / 2,-3 / 2,2,3,-2,1,1 / 2,1 / 3$. Note that it is reassuring that these special values come in pairs such as $(-1 / 5,-5)$. This is bound to be the case when we realize that the role of $x$ and $y$ should be interchangeable.

3. With a final example we wish to illustrate another benefit which the extra freedom incorporated in our generalization can offer. The point is that the form of the given family of curves may be suggestive for selecting a multiplier $g$ which will facilitate the computation of a corresponding potential. Consider a family of conics represented by

$$
f(x, y)=\frac{1}{2} a x^{2}+\frac{1}{2} b y^{2}+k x, \quad a, b, k \text { constant } .
$$

We have that

$$
\Delta:=2 f_{x} f_{y} f_{x y}-f_{x}^{2} f_{y y}-f_{y}^{2} f_{x x}=-2 a b f-b k^{2} .
$$


This is a numerator in the coefficient of $V$ in the generalized Szebehely equation (39). But there is also a denominator in that coefficient and it so happens that if we choose

$$
g_{11}=a, \quad g_{12}=0, \quad g_{22}=b,
$$

we get that

$$
g_{11} f_{y}^{2}-2 g_{12} f_{x} f_{y}+g_{22} f_{x}^{2}=-\Delta,
$$

which clearly considerably simplifies (39). The equation reduces to

$$
(a x+k) \frac{\partial V}{\partial x}+a y \frac{\partial V}{\partial y}=2 a(\bar{E}(f)-V)
$$

where $\bar{E}$ so far is an arbitrary function of $f$. Using the method of characteristics, we find for the homogeneous part that $y=c_{1}(a x+k)$ upon which the remaining characteristic equation can be written in the form

$$
\frac{d V}{d x}+\frac{2 a}{a x+k} V=\frac{2 a}{a x+k} \bar{E}(\tilde{f})
$$

where $\tilde{f}(x):=f\left(x, c_{1}(a x+k)\right)$. The solution of this equation is given by

$$
V=\frac{1}{(a x+k)^{2}}\left[c_{2}+H\left(x, c_{1}\right)\right], \quad \text { with } \quad H\left(x, c_{1}\right)=\int 2 a(a x+k) \bar{E}(\tilde{f}) d x .
$$

It then follows that the general solution for the Szebehely type equation is given by

$$
V(x, y)=\frac{1}{(a x+k)^{2}} F\left(\frac{y}{a x+k}\right)+\frac{1}{(a x+k)^{2}} H\left(x, \frac{y}{a x+k}\right),
$$

where $F$ is an arbitrary function of the indicated argument. To obtain a more explicit solution, let us make a choice for the function $\bar{E}$, say $\bar{E}(f):=f$. The function $H\left(x, c_{1}\right)$ can then readily be computed and we find that in the end

$$
V(x, y)=\frac{1}{(a x+k)^{2}} F\left(\frac{y}{a x+k}\right)+\frac{1}{2} f(x, y)-\frac{k^{2}}{4 a} .
$$

Note that the additive constant in $V$ can of course be omitted, but that has a similar effect on the corresponding energy function $\bar{E}$.

We take this opportunity now to test the consistency of the different approaches discussed in the previous section. The equation (41) for $\eta$ takes the simple form

$$
Z_{0}^{\perp}(\eta)+4 a b \eta=2 \bar{E}^{\prime}(f)
$$

and with our choice for $\bar{E}$ it explicitly reads

$$
(a x+k) \frac{\partial \eta}{\partial x}+a y \frac{\partial \eta}{\partial y}=-4 a \eta+\frac{2}{b} .
$$


The general solution of this equation is found to be

$$
\eta(x, y)=\frac{1}{(a x+k)^{4}} G\left(\frac{y}{a x+k}\right)+\frac{1}{2 a b},
$$

where $G$ again is an arbitrary function, which should however be related to $F$ in one way or another. We can now proceed as follows. With this expression for $\eta$, the relations (18, (19) provide admissible forces which, in accordance with our choice for the multiplier $g$, should satisfy the relation (cf. (29)) $b Y_{x}-a X_{y}=0$. It is straightforward to verify that this is indeed the case and it implies that we should have (in agreement with (30, 31) ):

$$
a X=-V_{x}, \quad b Y=-V_{y} .
$$

Since we have computed $V$ in a different way first, these equations should give us the relation between the arbitrary functions $F$ and $G$. Putting $z=y /(a x+k)$, this relation is found to be

$$
F(z)=-\frac{1}{2} b\left(1+a b z^{2}\right) G(z) .
$$

\section{Conclusions}

We have introduced a new element into an old problem, which essentially consists of combining ideas of two different but not completely unrelated inverse problems. We have argued that there is a clear distinction between the planar situation $(n=2)$ discussed in the present paper and the problem for $n>2$. We will come back to the case of higher dimension in a forthcoming paper. Where possible, we have been cautious about introducing basic concepts and using notations which make sense also for arbitrary dimension (see for example the very start of the analysis with conditions (10)). It should also be clear, from the way we formulated equations such as (38) and (40), that geometrical aspects will become increasingly important when we move to higher dimensions.

\section{Acknowledgements}

This work is part of the IRSES project GEOMECH (nr. 246981) within the 7th European Community Framework Programme. W. Sarlet and T. Mestdag thank the Australian Mathematical Sciences Institute for its hospitality. G. Prince and W. Sarlet further are indebted to Olga Rossi for useful discussions and for support from the Czech Science Foundation under grant No 201/09/0981. We are indebted to a referee for bringing the work of Kasner to our attention.

\section{Appendix}

What follows is a survey of the continuation of the analysis about the curves $x y^{m}=c$, as initiated in Section 5. 
Case 2 also requires Case c and with the conditions (43), we will have

$$
\begin{gathered}
m=-1 / 4, \quad r_{4}=0, \quad a_{1}=b_{1}=0, \quad s_{3} \text { so far arbitrary } \\
r_{1}=(32 / 5) b_{2}, \quad r_{2}=(52 / 7) b_{3}, \quad r_{3}=10 b_{4}, \quad s_{1}=8 a_{2}, \quad s_{2}=12 a_{3} .
\end{gathered}
$$

The condition (29) again produces five more requirements. With $g_{12}=0$, and then $g_{22}=1$ as before, we get that $a_{3}=b_{3}=0$ while $b_{4}, b_{2}, a_{2}$ remain free and finally: $s_{3}=5\left(b_{4} a_{2} / b_{2}\right)$ and $g_{11}=10\left(b_{4} / b_{2}\right)$. The potential is

$$
V(x, y)=-\frac{8}{5} b_{2} y^{4}-5 b_{4} x^{2} y^{2}-\frac{5}{2} \frac{b_{4}^{2}}{b_{2}} x^{4}-\frac{8}{3} a_{2} y^{3}-5 \frac{b_{4} a_{2}}{b_{2}} x^{2} y .
$$

If, on the other hand, we allow for a non-diagonal multiplier, with $g_{12}=1$, then $b_{2}, a_{2}, a_{3}$ remain arbitrary and the more exotic restrictions on the other parameters read

$$
b_{3}=(49 / 25)\left(b_{2} a_{3} / a_{2}\right), \quad b_{4}=(98 / 125)\left(b_{2} a_{3}^{2} / a_{2}^{2}\right), \quad s_{3}=(72 / 25)\left(a_{3}^{2} / a_{2}\right),
$$

with $g_{11}=-(14 / 5)\left(a_{3} / a_{2}\right)$ and $g_{22}=(5 / 4)\left(a_{2} / a_{3}\right)$. The reader can verify that the corresponding potential has five quartic and four cubic terms.

When we step into Case 3, there are clearly three subcases to consider. In Case 3a, the immediate restrictions are

$$
\begin{gathered}
m=-5, \quad r_{4}=s_{3}=0, \quad a_{1}=0, \quad b_{1} \text { so far arbitrary } \\
r_{1}=(1 / 15) b_{2}, \quad r_{2}=(1 / 10) b_{3}, \quad r_{3}=(3 / 25) b_{4}, \quad s_{1}=(3 / 55) a_{2}, \quad s_{2}=(7 / 75) a_{3} .
\end{gathered}
$$

For the restrictions coming from the search for a potential, if we first go for a diagonal $g$ again, so $g_{12}=0$ and $g_{22}=1$, we find that $a_{2}=a_{3}=b_{3}=b_{1}=0, b_{2}$ and $b_{4}$ remain arbitrary and $g_{11}=(3 / 25)\left(b_{4} / b_{2}\right)$. The potential is

$$
V(x, y)=-\frac{1}{60} b_{2} y^{4}-\frac{3}{50} b_{4} x^{2} y^{2}-\frac{3}{100} \frac{b_{4}^{2}}{b_{2}} x^{4} .
$$

For a non-diagonal $g$, with $g_{12}=1$, the coefficients become rather exotic again, so we limit ourselves to stating that $b_{1}, a_{2}$ and $a_{3}$ can be freely chosen; $b_{2}, b_{3}, b_{4}$ and also $g_{11}, g_{22}$ are subsequently fixed.

In Case 3b, the restrictions are

$$
\begin{gathered}
m=-4, \quad r_{4}=s_{3}=0, \quad b_{1}=0, \quad a_{1} \text { so far arbitrary } \\
r_{1}=(1 / 10) b_{2}, \quad r_{2}=(7 / 52) b_{3}, \quad r_{3}=(5 / 32) b_{4}, \quad s_{1}=(1 / 12) a_{2}, \quad s_{2}=(1 / 8) a_{3} .
\end{gathered}
$$

For a diagonal $g$, with $g_{12}=0$ and $g_{22}=1$, we end up with $a_{2}=b_{3}=0, a_{1}, a_{3}, b_{2}$ can be left free to choose, upon which $b_{4}=(2 / 5)\left(a_{3} b_{2} / a_{1}\right)$ and $g_{11}=(1 / 16)\left(a_{3} / a_{1}\right)$. The potential reads

$$
V(x, y)=-\frac{1}{40} b_{2} y^{4}-\frac{1}{32} \frac{a_{3} b_{2}}{a_{1}} y^{2} x^{2}-\frac{1}{160} \frac{a_{3}^{2} b_{2}}{a_{1}^{2}} x^{4}-\frac{1}{16} a_{3} y^{2} x-\frac{1}{48} \frac{a_{3}^{2}}{a_{1}} x^{3} .
$$


With $g_{12}=1$ on the other hand $a_{2}$ and $b_{3}$ need not be zero; the situation then is that $a_{1}, a_{2}, b_{2}$ can be left arbitrary, resulting in fixed expressions for $a_{3}, b_{3}, b_{4}$ as well as $g_{11}$ and $g_{22}$. But as before, listing the corresponding potential is not very instructive in this case because of exotic coefficients.

Finally, we look at the more interesting Case 3c where $m$ is not immediately fixed. We have $r_{4}=s_{3}=a_{1}=b_{1}=0$ and from (43) generically,

$$
\begin{gathered}
r_{1}=\frac{2}{m(m-1)} b_{2}, \quad r_{2}=\frac{3-m}{m(3 m-1)} b_{3}, \quad r_{3}=\frac{1-m}{2 m^{2}} b_{4}, \\
s_{1}=\frac{3}{m(2 m-1)} a_{2}, \quad s_{2}=\frac{2-m}{3 m^{2}} a_{3} .
\end{gathered}
$$

Again, there are three special values which will have to be discussed separately (cf. [9]): $m=1, m=1 / 2$ and $m=1 / 3$. We will do so further on. Continuing first with the generic case and looking at the five extra identifications coming from the inverse problem condition (29), we first consider the possibility of a diagonal $g$ again, with $g_{12}=0$ and $g_{22}=1$. It follows that $a_{2}=b_{3}=0$ and either $a_{3}=0$ or $m=2$. This is in fact the situation described in [9], but since our diagonal $g$ need not be the unit matrix, we see that even here a somewhat more general solution appears, namely (with $m$ arbitrary for the moment) $b_{2}$ and $b_{4}$ can both be left arbitrary, it suffices to adjust the multiplier by taking

$$
g_{11}=\frac{1-m}{2 m^{2}} \frac{b_{4}}{b_{2}}
$$

The potential is given by

$$
V(x, y)=-\frac{1}{2} \frac{b_{2}}{m(m-1)} y^{4}+\frac{1}{4} \frac{(m-1) b_{4}}{m^{2}} x^{2} y^{2}+\frac{1}{8} \frac{(m-1) b_{4}^{2}}{m^{2} b_{2}} x^{4} .
$$

When we look for a non-diagonal $g$ with $g_{12}=1$, the five identifications coming from (29) can be written as follows,

$$
\begin{aligned}
a_{2} g_{11} & =a_{3} \frac{(3 m+2)(2 m-1)}{3 m^{2}}, \\
b_{3} g_{11} & =b_{4} \frac{(3 m-1)(2 m+1)}{2 m^{2}} \\
a_{3} g_{22} \frac{2-m}{3 m^{2}} & =a_{2} \frac{(2 m+3)(2-m)}{m(2 m-1)} \\
b_{3} g_{22} \frac{3-m}{m(3 m-1)} & =b_{2} \frac{(3-m)(2+m)}{m(m-1)} \\
b_{4} g_{22} \frac{1-m}{m^{2}}-2 b_{2} g_{11} & =6 b_{3} \frac{(1-m)(1+m)}{m(3 m-1)} .
\end{aligned}
$$

This leads to a quite extensive list of subcases to be considered. In particular, various new special values for $m$ present themselves, namely $m=-2 / 3, m=-1 / 2, m=-3 / 2$, $m=2$ again, $m=3$ and $m=-2$. For all these values a $g$ and corresponding potential 
can be found; often of course, it will lead also to a number of the $b_{i}$ and $a_{i}$ having to be zero, so that the forces will contain only a couple of terms. We limit ourselves to further cases in which $m$ can be left unspecified. No such cases occur when we take either $g_{11}=0$ or $g_{22}=0$. Insisting that both should be nonzero, if also $a_{2}$ and $a_{3}$ are nonzero, they are determined by the first and third condition (44). To avoid a conflict with the excluded value $m=-1$, we must have $b_{3}=0$ and if no specification of $m$ is permitted, it further follows that $b_{4}=b_{2}=0$. In the end, the $a_{i}$ and $m$ are still arbitrary, so we are left with only quadratic forces a nd a potential which is given by

$$
V(x, y)=-3 \frac{(2 m+3) a_{2}^{2}}{(2 m-1)^{2} a_{3}} y^{3}-a_{3} x^{2} y-3 \frac{a_{2}}{m(2 m-1)} x y^{2}-\frac{1}{9} \frac{(2 m-1)(3 m+2) a_{3}^{2}}{m^{2} a_{2}} x^{3} .
$$

If on the other hand $a_{2}=a_{3}=0$, then $b_{3}$ cannot be zero for general $m$ and $g_{11}, g_{22}$ are determined by the second and fourth of the relations (44). The remaining fifth condition imposes the following relation between the $b_{i}$,

$$
2 m(1-m) b_{3}^{2}+(3 m-1)^{2} b_{2} b_{4}=0
$$

The conclusion here is that $m$ is still arbitrary, that we have forces with only cubic terms this time and the potential is given by

$$
\begin{aligned}
V(x, y)= & -2 \frac{m(m+2) b_{3}^{3}}{(3 m-1)^{3} b_{4}^{2}} y^{4}-4 \frac{b_{3}^{2}}{(3 m-1)^{2} b_{4}} y^{3} x \\
& -\frac{\left(m^{2}-m+1\right) b_{3}}{m(3 m-1)} y^{2} x^{2}-b_{4} y x^{3}-\frac{1}{8} \frac{(2 m+1)(3 m-1) b_{4}^{2}}{m^{2} b_{3}} x^{4} .
\end{aligned}
$$

It remains to discuss, still for Case $3 \mathrm{c}$, the three special values of $m$ which were distinguished before imposing the inverse problem condition and for which no potential was found in [8].

For $m=1$, the analysis is straightforward and the conclusions go as follows. Remembering that $r_{4}=s_{3}=a_{1}=b_{1}=0$ already, we also have $b_{2}=0$ now. For a diagonal $g$ with $g_{22}=1$, we further must have $b_{3}=a_{2}=a_{3}=0$ and the potential is given by

$$
V(x, y)=-\frac{1}{4}\left(r_{1} y^{4}+g_{11} b_{4} x^{4}\right) .
$$

The fact that $g_{11}$ is unspecified here is the result of a decoupling of the equations (and it reflects the symmetry between $x$ and $y$ in this case). For a non-diagonal $g, a_{2}, a_{3}$ and $b_{3}$ can be left arbitrary, then $b_{4}=(5 / 9)\left(a_{3} b_{3} / a_{2}\right), r_{1}=5\left(a_{2} b_{3} / a_{3}\right)$ and with $g_{12}=1$, $g_{11}=(5 / 3)\left(a_{3} / a_{2}\right)$ and $g_{22}=15\left(a_{2} / a_{3}\right)$. The corresponding potential is

$$
\begin{aligned}
V(x, y)= & -\frac{75}{4} \frac{a_{2}^{2} b_{3}}{a_{3}^{2}} y^{4}-5 \frac{a_{2} b_{3}}{a_{3}} y^{3} x-\frac{1}{2} b_{3} y^{2} x^{2}-\frac{5}{9} \frac{a_{3} b_{3}}{a_{2}} y x^{3} \\
& -\frac{25}{108} \frac{a_{3}^{2} b_{3}}{a_{2}^{2}} x^{4}-15 \frac{a_{2}^{2}}{a_{3}} y^{3}-3 a_{2} y^{2} x-a_{3} y x^{2}-\frac{5}{9} \frac{a_{3}^{2}}{a_{2}} x^{3} .
\end{aligned}
$$


Likewise, for $m=1 / 2, a_{2}=0$ and for a diagonal $g$ with $g_{22}=1$, also $a_{3}=b_{3}=0$ while $g_{11}=b_{4} / b_{2}$. The potential is

$$
V(x, y)=2 b_{2} y^{4}-\frac{1}{2} b_{4} y^{2} x^{2}-\frac{1}{4} \frac{b_{4}^{2}}{b_{2}} x^{4}-\frac{1}{3} s_{1} y^{3} .
$$

For a non-diagonal $g$ on the other hand, $a_{3}, b_{2}$ and $b_{3}$ can be seen as arbitrary, then $b_{4}=-2\left(b_{3}^{2} / b_{2}\right), s_{1}=-(5 / 2)\left(b_{2} a_{3} / b_{3}\right), g_{11}=-4\left(b_{3} / b_{2}\right), g_{22}=-(5 / 2)\left(b_{2} / b_{3}\right)$. The corresponding potential can easily be computed. We leave the final case $m=1 / 3$ to the reader.

\section{References}

[1] J. E. Aldridge, G. E. Prince, W. Sarlet and G. Thompson, An EDS approach to the inverse problem in the calculus of variations, J. Math. Phys. 47 (2006) 103508.

[2] M-C. Anisiu, Two- and three-dimensional inverse problem of dynamics, Studia Univ. Babeş-Bolyai, Math. XLIX(4) (2004) 13-26.

[3] M-C. Anisiu, An alternative point of view on the equations of the inverse problem of dynamics, Inverse Problems 20 (2004) 1865-1872.

[4] I. Anderson and G. Thompson, The inverse problem of the calculus of variations for ordinary differential equations, Mem. Amer. Math. Soc. 473 (1992).

[5] F. Borghero, Variational determination of the generalized Szebehely's equations, Rend. Sem. Mat. Univers. Politecn. Torino 45 (1987) 125-135.

[6] G. Bozis, Inverse problem with two-parametric families of planar orbits, Celest. Mech. 31 (1983) 129-43.

[7] G. Bozis, Szebehely's inverse problem for finite symmetrical material concentrations, Astron. Astrophys. 134 (1984) 360-364.

[8] G. Bozis, Family boundary curves for autonomous dynamical systems, Celest. Mech. Dyn. Astron 60 (1994) 161-172.

[9] G. Bozis, The inverse problem of dynamics: basic facts, Inverse Problems 11 (1995) 687-708.

[10] G. Bozis and M-C. Anisiu, Families of straight lines in planar potentials, Rom. Astron. J. 11 (2001) 27-43.

[11] R. Broucke and H. Lass, On Szebehely's Equation for the Potential of a Prescribed Family of Orbits, Celest. Mech. 16 (1977) 215-225.

[12] I. Bucataru and M.F. Dahl, Semi-basic 1-forms and Helmholtz conditions for the inverse problem of the calculus of variations, J. Geom. Mech. 1 (2009) 159-180. 
[13] J.F. Cariñena, X. Gràcia, G. Marmo, E. Martínez, M.C. Muñoz-Lecanda, N. RománRoy, Geometric Hamilton-Jacobi theory, Int. J. Geom. Meth. Mod. Phys. 3 (2006) $1417-1458$.

[14] M. Crampin, W. Sarlet, E. Martínez, G.B. Byrnes and G.E. Prince, Towards a geometrical understanding of Douglas's solution of the inverse problem of the calculus of variations, Inverse problems 10 (1994) 245-260.

[15] M. Crampin and F.A.E. Pirani, Applicable Differential Geometry, London Math. Soc. Lecture Note Series 59 (Cambridge University Press) (1987).

[16] U. Dainelli, Sul movimento per una linea qualunque, Giornale di Matematiche (Napoli) XVIII (1880) 271-300.

[17] F. Gonzales-Gascon, A. Gonzales-Lopez and P.J. Pascal-Broncano, On Szebehely's equation and its connection with Dainelly's-Whittaker's equations, Celest. Mech. 33 (1984) 85-97.

[18] J. Grifone and Z. Muzsnay, On the inverse problem of the variational calculus: existence of Lagrangians associated with a spray in the isotropic case, Ann. Inst. Fourier 49 (1999) 1387-1421.

[19] E. Kasner, The trajectories of dynamics, Trans. Am. Math. Soc. 7 (1906) 401-424.

[20] E. Kasner, Natural families of trajectories: conservative fields of force, Trans. Am. Math. Soc. 10 (1909) 201-219.

[21] E. Kasner and J. DeCicco, A generalized theory of dynamical trajectories, Trans. Am. Math. Soc. 54 (1943) 23-38.

[22] O. Krupková and G. E. Prince, Second order ordinary differential equations in jet bundles and the inverse problem of the calculus of variations, in Handbook of Global Analysis D. Krupka and D. Saunders eds. (Elsevier 2008) 837-904.

[23] A. Melis and B. Piras, An extension of Szebehely's problem to holonomic systems, Celest. Mech. 32 (1984) 87-92.

[24] R. Mertens, On the determination of the potential energy of a particle describing orbits on a given surface, ZAMM 61 (1981) 252-253.

[25] S. Molnar, Applications of Szebehely's equation, Celest. Mech. 25 (1981) 81-88.

[26] G. Morandi, C. Ferrario, G. Lo Vecchio, G. Marmo and, C. Rubano, The inverse problem in the calculus of variations and the geometry of the tangent bundle, Physics Reports 188 Issues 3-4, (1990) 147-284.

[27] A. Pal and M.C. Anisiu, On the two-dimensional inverse problem of dynamics, Astron. Nachr. 317 (1996) 205-209. 
[28] F. Puel, Relation entre l'équation de Szebehely du problème inverse de la mécanique et la theorie de Hamilton-Jacobi, Celest. Mech. Dyn. Astron. 46 (1989) 31-34.

[29] F. Puel, Separable and partially separable systems in the light of the inverse problem of dynamics, Celest. Mech. Dyn. Astron. 63 (1995) 41-57.

[30] W. Sarlet, The Helmholtz conditions revisited. A new approach to the inverse problem of Lagrangian dynamics, J. Phys. A: Math. Gen. 15 (1982) 1503-1517.

[31] V. Szebehely, On the determination of the potential by satellite observations, Proc. Int. Meeting on Earths Rotations by Satellite Observations, Rend. Sem. Fac. Sc. Univ. of Cagliari, XLIV Suppl., E. Proverbio (ed), (Bologna: Pitagora Editrice) (1974) 31-35. 